\title{
Substitutions over infinite alphabet generating $(-\beta)$-integers
}

\author{
Daniel Dombek \\ Department of Mathematics FNSPE \\ Czech Technical University in Prague \\ Czech Republic \\ dombedan@fjfi.cvut.cz
}

\section{Introduction}

This contribution is devoted to the study of positional numeration systems with negative base introduced by Ito and Sadahiro in 2009 , called $(-\beta)$-expansions. We give an admissibility criterion for more general case of $(-\beta)$-expansions and discuss the properties of the set of $(-\beta)$-integers, denoted by $\mathbb{Z}_{-\beta}$. We give a description of distances within $\mathbb{Z}_{-\beta}$ and show that this set can be coded by an infinite word over an infinite alphabet, which is a fixed point of a non-erasing non-trivial morphism.

\section{Numeration with negative base}

In 1957, Rényi introduced positional numeration system with positive real base $\beta>1$ (see [7]). The $\beta$-expansion of $x \in[0,1)$ is defined as the digit string $d_{\beta}(x)=0 \bullet x_{1} x_{2} x_{3} \cdots$, where

$$
x_{i}=\left\lfloor\beta T_{\beta}^{i-1}(x)\right\rfloor \quad \text { and } \quad T_{\beta}(x)=\beta x-\lfloor\beta x\rfloor .
$$

It holds that

$$
x=\frac{x_{1}}{\beta}+\frac{x_{2}}{\beta^{2}}+\frac{x_{3}}{\beta^{3}}+\cdots .
$$

Note that this definition can be naturally extended so that any real number has a unique $\beta$-expansion, which is usually denoted $d_{\beta}(x)=x_{k} x_{k-1} \cdots x_{1} x_{0} \bullet x_{-1} x_{-2} \cdots$, where $\bullet$, the fractional point, separates negative and non-negative powers of $\beta$. In analogy with standard integer base, the set $\mathbb{Z}_{\beta}$ of $\beta$-integers is defined as the set of real numbers having the $\beta$-expansion of the form $d_{\beta}(x)=x_{k} x_{k-1} \cdots x_{1} x_{0} \bullet 0^{\omega}$.

$(-\beta)$-expansions, a numeration system built in analogy with Rényi $\beta$-expansions, was introduced in 2009 by Ito and Sadahiro (see [5]). They gave a lexicographic criterion for deciding whether some digit string is the $(-\beta)$-expansion of some $x$ and also described several properties of $(-\beta)$-expansions concerning symbolic dynamics and ergodic theory. Note that dynamical properties of $(-\beta)$-expansions were also studied by Frougny and Lai (see [4]). We take the liberty of defining $(-\beta)$-expansions in a more general way, while an analogy with positive base numeration can still be easily seen.

Definition 1. Let $-\beta<-1$ be a base and consider $x \in[l, l+1)$, where $l \in \mathbb{R}$ is arbitrary fixed. We define the $(-\beta)$-expansion of $x$ as the digit string $d(x)=x_{1} x_{2} x_{3} \cdots$, with digits $x_{i}$ given by

$$
x_{i}=\left\lfloor-\beta T^{i-1}(x)-l\right\rfloor,
$$

where $T(x)$ stands for the generalised $(-\beta)$-transformation

$$
T:[l, l+1) \rightarrow[l, l+1), \quad T(x)=-\beta x-\lfloor-\beta x-l\rfloor .
$$

P. Ambrož, Š. Holub and Z. Masáková (Eds.):

8th International Conference WORDS 2011

EPTCS 63, 2011, pp. 115-121 doi 10.4204/EPTCS.63.16 
It holds that

$$
x=\frac{x_{1}}{-\beta}+\frac{x_{2}}{(-\beta)^{2}}+\frac{x_{3}}{(-\beta)^{3}}+\cdots
$$

and the fractional point is again used in the notation, $d(x)=0 \bullet x_{1} x_{2} x_{3} \cdots$.

The set of digits used in $(-\beta)$-expansions of numbers (in the latter referred to as the alphabet of $(-\beta)$-expansions) depends on the choice of $l$ and can be calculated directly from (1) as

$$
\mathscr{A}_{-\beta, l}=\{\lfloor-l(\beta+1)-\beta\rfloor, \ldots,\lfloor-l(\beta+1)\rfloor\} .
$$

We may demand that the numeration system possesses various properties. Let us summarise the most natural ones:

- The most common requirement is that zero is an allowed digit. We see that $0 \in \mathscr{A}_{-\beta, l}$ is equivalent to $0 \in[l, l+1)$ and consequently $l \in(-1,0]$. Note that this implies $d(0)=0 \bullet 0^{\omega}$.

- We may require that $\mathscr{A}_{-\beta, l}=\{0,1, \ldots,\lfloor\beta\rfloor\}$. This is equivalent to the choice $l \in\left(-\frac{\lfloor\beta\rfloor+1}{\beta+1},-\frac{\beta}{\beta+1}\right]$.

- So far, $(-\beta)$-expansions were defined only for numbers from $[l, l+1)$. In Rényi numeration, the $\beta$-expansion of arbitrary $x \in \mathbb{R}^{+}$(expansions of negative numbers differ only by "-" sign) is defined as $d_{\beta}(x)=x_{k} x_{k-1} \cdots x_{1} x_{0} \bullet x_{-1} x_{-2} \cdots$, where $k \in \mathbb{N}$ satisfies $\frac{x}{\beta^{k}} \in[l, l+1)$ and $d_{\beta}\left(\frac{x}{\beta^{k}}\right)=$ $0 \bullet x_{k} x_{k-1} x_{k-2} \cdots$. The same procedure does not work for $(-\beta)$-expansions in general. A necessary and sufficient condition for the existence of unique $d(x)$ for all $x \in \mathbb{R}$ is that $-\frac{1}{\beta}[l, l+1) \subset[l, l+1)$. This is equivalent to the choice $l \in\left(-\frac{\beta}{\beta+1},-\frac{1}{\beta+1}\right]$. Note that this choice is disjoint with the previous one, so one cannot have uniqueness of $(-\beta)$-expansions and non-negative digits bounded by $\beta$ at the same time.

Let us stress that in the following we will need 0 to be a valid digit. Therefore, we shall always assume $l \in(-1,0]$. Note that we may easily derive that the digits in the alphabet $\mathscr{A}_{-\beta, l}$ are then bounded by $\lceil\beta\rceil$ in modulus.

\section{Admissibility}

In Rényi numeration there is a natural correspondence between ordering on real numbers and lexicographic ordering on their $\beta$-expansions. In $(-\beta)$-expansions, standard lexicographic ordering is not suitable anymore, hence a different ordering on digit strings is needed.

The so-called alternate order was used in the admissibility condition by Ito and Sadahiro and it will work also in the general case. Let us recall the definition. For the strings

$$
u, v \in\left(\mathscr{A}_{-\beta, l}\right)^{\mathbb{N}}, \quad u=u_{1} u_{2} u_{3} \cdots \quad \text { and } \quad v=v_{1} v_{2} v_{3} \cdots
$$

we say that $u \prec_{\text {alt }} v$ ( $u$ is less than $v$ in the alternate order) if $u_{m}(-1)^{m}<v_{m}(-1)^{m}$, where $m=\min \{k \in$ $\left.\mathbb{N} \mid u_{k} \neq v_{k}\right\}$. Note that standard ordering between reals in $[l, l+1)$ corresponds to the alternate order on their respective $(-\beta)$-expansions.

Definition 2. An infinite string $x_{1} x_{2} x_{3} \cdots$ of integers is called $(-\beta)$-admissible (or just admissible), if there exists an $x \in[l, l+1)$ such that $x_{1} x_{2} x_{3} \cdots$ is its $(-\beta)$-expansion, i.e. $x_{1} x_{2} x_{3} \cdots=d(x)$.

We give the criterion for $(-\beta)$-admissibility (proven in [2]) in a form similar to both Parry lexicographic condition (see [6]) and Ito-Sadahiro admissibility criterion (see [5]). 
Theorem 3. ([2]) An infinite string $x_{1} x_{2} x_{3} \cdots$ of integers is $(-\beta)$-admissible, if and only if

$$
l_{1} l_{2} l_{3} \cdots \preceq_{\text {alt }} x_{i} x_{i+1} x_{i+2} \cdots \prec_{\text {alt }} r_{1} r_{2} r_{3} \cdots, \quad \text { for all } i \geq 1,
$$

where $l_{1} l_{2} l_{3} \cdots=d(l)$ and $r_{1} r_{2} r_{3} \cdots=d^{*}(l+1)=\lim _{\varepsilon \rightarrow 0+} d(l+1-\varepsilon)$.

Remark 4. Ito and Sadahiro have described the admissibility condition for their numeration system considered with $l=-\frac{\beta}{\beta+1}$. This choice imply for any $\beta$ the alphabet of the form $\mathscr{A}_{-\beta, l}=\{0,1, \ldots,\lfloor\beta\rfloor\}$. They have shown that in this case the reference strings used in the condition in Theorem 3 (i.e. $d(l)=$ $l_{1} l_{2} l_{3} \cdots$ and $\left.d^{*}(l+1)=r_{1} r_{2} r_{3} \cdots\right)$ are related in the following way:

$$
r_{1} r_{2} r_{3} \cdots=0 l_{1} l_{2} l_{3} \cdots
$$

if $d(l)$ is not purely periodic with odd period length, and,

$$
r_{1} r_{2} r_{3} \cdots=\left(0 l_{1} l_{2} \cdots l_{q-1}\left(l_{q}-1\right)\right)^{\omega}
$$

if $d(l)=\left(l_{1} l_{2} \cdots l_{q}\right)^{\omega}$, where $q$ is odd.

Remark 5. Besides Ito-Sadahiro case and the general one, we may consider another interesting example, the choice $l=-\frac{1}{2}, \beta \notin 2 \mathbb{Z}+1$. This leads to a numeration defined on "almost symmetric" interval $\left[-\frac{1}{2}, \frac{1}{2}\right)$ with symmetric alphabet

$$
\mathscr{A}_{-\beta,-\frac{1}{2}}=\left\{\overline{\left\lfloor\frac{\beta+1}{2}\right\rfloor}, \ldots, \overline{1}, 0,1, \ldots\left\lfloor\frac{\beta+1}{2}\right\rfloor\right\} .
$$

Note that we use the notation $(-a)=\bar{a}$ for shorter writing of negative digits. If we denote the reference strings as usual, i.e. $d\left(-\frac{1}{2}\right)=l_{1} l_{2} l_{3} \cdots$ and $d^{*}\left(\frac{1}{2}\right)=r_{1} r_{2} r_{3} \cdots$, the following relation can be shown:

$$
r_{1} r_{2} r_{3} \cdots=\overline{l_{1} l_{2} l_{3} \cdots}
$$

if $d(l)$ is not purely periodic with odd period length, and,

$$
r_{1} r_{2} r_{3} \cdots=\left(\overline{l_{1} l_{2} \cdots l_{q-1}\left(l_{q}-1\right)} l_{1} l_{2} \cdots l_{q-1}\left(l_{q}-1\right)\right)^{\omega}
$$

if $d(l)=\left(l_{1} l_{2} \cdots l_{q}\right)^{\omega}$, where $q$ is odd.

\section{$4(-\beta)$-integers}

We have already discussed basic properties of $(-\beta)$-expansions and the question of admissibility of digit strings. In the following, $(-\beta)$-admissibility will be used to define the set of $(-\beta)$-integers.

Let us define a "value function" $\gamma$. Consider a finite digit string $x_{k-1} \cdots x_{1} x_{0}$, then $\gamma\left(x_{k-1}, \cdots x_{1} x_{0}\right)=$ $\sum_{i=0}^{k-1} x_{i}(-\beta)^{i}$.

Definition 6. We call $x \in \mathbb{R} a(-\beta)$-integer, if there exists $a(-\beta)$-admissible digit string $x_{k} x_{k-1} \cdots x_{0} 0^{\omega}$ such that $d(x)=x_{k} x_{k-1} \cdots x_{1} x_{0} \bullet 0^{\omega}$. The set of $(-\beta)$-integers is then defined as

$$
\mathbb{Z}_{-\beta}=\left\{x \in \mathbb{R} \mid x=\gamma\left(a_{k-1} a_{k-2} \cdots a_{1} a_{0}\right), a_{k-1} a_{k-2} \cdots a_{1} a_{0} 0^{\omega} \text { is }(-\beta) \text {-admissible },\right.
$$

or equivalently

$$
\mathbb{Z}_{-\beta}=\bigcup_{i \geq 0}(-\beta)^{i} T^{-i}(0)
$$


Note that $(-\beta)$-expansions of real numbers are not necessarily unique. As was said before, uniqueness holds if and only if $l \in\left(-\frac{\beta}{\beta+1},-\frac{1}{\beta+1}\right]$. Let us demonstrate this ambiguity on the following example.

Example 7. Let $\beta$ be the greater root of the polynomial $x^{2}-2 x-1$, i.e. $\beta=1+\sqrt{2}$, and let $[l, l+1)=$ $\left[-\frac{\beta^{9}}{\beta^{9}+1}, \frac{1}{\beta^{9}+1}\right)$. Note that $[l, l+1)$ is not invariant under division by $(-\beta)$.

If we want to find the $(-\beta)$-expansion of number $x \notin[l, l+1)$, we have to find such $k \in \mathbb{N}$ that $\frac{x}{(-\beta)^{k}} \in[l, l+1)$, compute $d\left(\frac{x}{(-\beta)^{k}}\right)$ by definition and then shift the fractional point by $k$ positions to the right. The problem is that, in general, different choices of the exponent $k$ may give different $(-\beta)$ admissible digit strings which all represent the same number $x$.

Let us find possible $(-\beta)$-expansions of 1 . It can be shown that $\frac{1}{(-\beta)^{k}} \in[l, l+1)$ if and only if $k \in \mathbb{N} \backslash\{0,2,4,6,8\}$ and there are $5(-\beta)$-admissible digit strings representing 1 , computed from (- $\beta)$ expansions of $\frac{1}{(-\beta)^{k}}$ for $k=1,3,5,7,9$ respectively:

$$
1 \bullet 0^{\omega}=120 \bullet 0^{\omega}=13210 \bullet 0^{\omega}=1322210 \bullet 0^{\omega}=132222210 \bullet 0^{\omega} \text {. }
$$

Let us mention some straightforward observations on the properties of $\mathbb{Z}_{-\beta}$ :

- $\mathbb{Z}_{-\beta}$ is nonempty if and only if $0 \in \mathscr{A}_{-\beta, l}$, i.e. if and only if $l \in(-1,0]$.

- The definition implies $-\beta \mathbb{Z}_{-\beta} \subset \mathbb{Z}_{-\beta}$.

- A phenomenon unseen in Rényi numeration arises, there are cases when the set of $(-\beta)$-integers is trivial, i.e. when $\mathbb{Z}_{-\beta}=\{0\}$. This happens if and only if both numbers $\frac{1}{\beta}$ and $-\frac{1}{\beta}$ are outside of the interval $[l, l+1)$. This can be reformulated as

$$
\mathbb{Z}_{-\beta}=\{0\} \quad \Leftrightarrow \quad \beta<-\frac{1}{l} \text { and } \beta \leq \frac{1}{l+1},
$$

and it can be seen that the strictest limitation for $\beta$ arises when $l=-\frac{1}{2}$. This implies for any choice of $l \in \mathbb{R}$ :

$$
\mathbb{Z}_{-\beta} \neq \emptyset \text { and } \beta \geq 2 \quad \Rightarrow \quad \mathbb{Z}_{-\beta} \supsetneq\{0\}
$$

- It holds that $\mathbb{Z}_{-\beta}=\mathbb{Z}$ if and only if $\beta \in \mathbb{N}$.

Remark 8. As was shown in Example 7 in a completely general case of (- $\beta)$-expansions, there is a problem with ambiguity. Because of this, in the following we shall limit ourselves to the choice $l \in[-$ $\left.\frac{\beta}{\beta+1},-\frac{1}{\beta+1}\right]$. Note that we allow Ito-Sadahiro case $l=-\frac{\beta}{\beta+1}$, which also contains ambiguities, but only in countably many cases, which can be avoided by introducing a notion of strong (- $\beta)$-admissibility.

Definition 9. Let $x_{1} x_{2} x_{3} \cdots \in \mathscr{A}_{-\beta, l}$. We say that

$$
x_{1} x_{2} x_{3} \cdots \text { is strongly }(-\beta) \text {-admissible if } 0 x_{1} x_{2} x_{3} \cdots \text { is }(-\beta) \text {-admissible. }
$$

Remark 10. Note that if $l \in\left(-\frac{\beta}{\beta+1},-\frac{1}{\beta+1}\right]$, the notions of strong admissibility and admissibility coincide. In the case $l=-\frac{\beta}{\beta+1}$, the only numbers with non-unique expansions are those of the form $(-\beta)^{k} l$, which have exactly two possible expansions using digit strings $l_{1} l_{2} l_{3} \cdots$ and $1 l_{1} l_{2} l_{3} \cdots$. While both are $(-\beta)$-admissible, only the latter is also strongly $(-\beta)$-admissible. 
In order to describe distances between adjacent $(-\beta)$-integers, we will study ordering of finite digit strings in the alternate order. Denote by $\mathscr{S}(k)$ the set of infinite $(-\beta)$-admissible digit strings such that erasing a prefix of length $k$ yields $0^{\omega}$, i.e. for $k \geq 0$, we have

$$
\mathscr{S}(k)=\left\{a_{k-1} a_{k-2} \cdots a_{0} 0^{\omega} \mid a_{k-1} a_{k-2} \cdots a_{0} 0^{\omega} \text { is }(-\beta) \text {-admissible }\right\},
$$

in particular $\mathscr{S}(0)=\left\{0^{\omega}\right\}$. For a fixed $k$, the set $\mathscr{S}(k)$ is finite. Denote by $\operatorname{Max}(k)$ the string $a_{k-1} a_{k-2} \cdots a_{0} 0^{\omega}$ which is maximal in $\mathscr{S}(k)$ with respect to the alternate order and by $\max (k)$ its prefix of length $k$, i.e. $\operatorname{Max}(k)=\max (k) 0^{\omega}$. Similarly, we define $\operatorname{Min}(k)$ and $\min (k)$. Thus,

$$
\operatorname{Min}(k) \preceq_{\text {alt }} r \preceq \text { alt } \operatorname{Max}(k), \quad \text { for all digit strings } r \in \mathscr{S}(k) .
$$

With this notation we can give a theorem describing distances in $\mathbb{Z}_{-\beta}$ valid for cases $l \in\left[-\frac{\beta}{\beta+1},-\frac{1}{\beta+1}\right]$. Note that for case $l=-\frac{\beta}{\beta+1}$ it was proven in [1].

Theorem 11. Let $x<y$ be two consecutive $(-\beta)$-integers. Then there exist a finite string $w$ over the alphabet $\mathscr{A}_{-\beta, l}$, a non-negative integer $k \in\{0,1,2, \ldots\}$ and a positive digit $d \in \mathscr{A}_{-\beta, l} \backslash\{0\}$ such that $w(d-1) \operatorname{Max}(k)$ and $w d \operatorname{Min}(k)$ are strongly $(-\beta)$-admissible strings and

$$
\begin{array}{lll}
x=\gamma(w(d-1) \max (k)) & <y=\gamma(w d \min (k)) & \text { for } k \text { even } \\
x=\gamma(w d \min (k)) & <y=\gamma(w(d-1) \max (k)) & \text { for } k \text { odd } .
\end{array}
$$

In particular, the distance $y-x$ between these $(-\beta)$-integers depends only on $k$ and equals to

$$
\Delta_{k}:=\left|(-\beta)^{k}+\gamma(\min (k))-\gamma(\max (k))\right| .
$$

\section{Coding $\mathbb{Z}_{-\beta}$ by an infinite word}

Note that in order to get an explicit formula for distances from Theorem 3 , knowledge of reference strings $\min (k)$ and $\max (k)$ is necessary. These depend on both reference strings $d(l)$ and $d^{*}(l+1)$. Concerning the form of $\min (k)$ and $\max (k)$ we provide the following proposition.

Proposition 12. Let $\beta>1$. Denote $d(l)=l_{1} l_{2} l_{3} \cdots, d^{*}(l+1)=r_{1} r_{2} r_{3} \cdots$.

- $\min (0)=\max (0)=\varepsilon$,

- for $k \geq 1$ either $\min (k)=l_{1} l_{2} \cdots l_{k}$ or there exists $m(k) \in\{0, \cdots, k-1\}$ such that

$$
\min (k)= \begin{cases}l_{1} l_{2} \cdots\left(l_{k-m(k)}+1\right) \min (m(k)) & \text { if } k-m(k) \text { even } \\ l_{1} l_{2} \cdots\left(l_{k-m(k)}-1\right) \max (m(k)) & \text { if } k-m(k) \text { odd }\end{cases}
$$

- for $k \geq 1$ either $\max (k)=r_{1} r_{2} \cdots r_{k}$ or there exists $m^{\prime}(k) \in\{0, \cdots, k-1\}$ such that

$$
\max (k)= \begin{cases}r_{1} r_{2} \cdots\left(r_{k-m^{\prime}(k)}-1\right) \max \left(m^{\prime}(k)\right) & \text { if } k-m^{\prime}(k) \text { even } \\ r_{1} r_{2} \cdots\left(r_{k-m^{\prime}(k)}+1\right) \min \left(m^{\prime}(k)\right) & \text { if } k-m^{\prime}(k) \text { odd }\end{cases}
$$


Computing $\min (k)$ and $\max (k)$ for a general choice of $l$ may lead to difficult discussion, however, in special cases an important relation between $d(l)$ and $d^{*}(l+1)$ arises and eases the computation. Examples were given in Remarks 4 and 5

Let us now describe how we can code the set of $(-\beta)$-integers by an infinite word over the infinite alphabet $\mathbb{N}$.

Let $\left(z_{n}\right)_{n \in \mathbb{Z}}$ be a strictly increasing sequence satisfying

$$
z_{0}=0 \quad \text { and } \quad \mathbb{Z}_{-\beta}=\left\{z_{n} \mid n \in \mathbb{Z}\right\}
$$

We define a bidirectional infinite word over an infinite alphabet $\mathbf{v}_{-\beta} \in \mathbb{N}^{\mathbb{Z}}$, which codes the set of $(-\beta)$ integers. According to Theorem 11 , for any $n \in \mathbb{Z}$ there exist a unique $k \in \mathbb{N}$, a word $w$ with prefix 0 and a letter $d$ such that

$$
z_{n+1}-z_{n}=|\gamma(w(d-1) \max (k))-\gamma(w d \min (k))| .
$$

We define the word $\mathbf{v}_{-\beta}=\left(v_{i}\right)_{i \in \mathbb{Z}}$ by $v_{n}=k$.

Theorem 13. Let $\mathbf{v}_{-\beta}$ be the word associated with $(-\beta)$-integers. There exists an antimorphism $\Phi$ : $\mathbb{N}^{*} \rightarrow \mathbb{N}^{*}$ such that $\Psi=\Phi^{2}$ is a non-erasing non-identical morphism and $\Psi\left(\mathbf{v}_{-\beta}\right)=\mathbf{v}_{-\beta}$. $\Phi$ is always of the form

$$
\Phi(2 l)=S_{2 l}(2 l+1) \widetilde{R_{2 l}} \text { and } \Phi(2 l+1)=R_{2 l+1}(2 l+2) \widetilde{S_{2 l+1}},
$$

where $\tilde{u}$ denotes the reversal of the word $u$ and words $R_{j}, S_{j}$ depend only on $j$ and on $\min (k), \max (k)$ with $k \in\{j, j+1\}$.

The proof is based on the self-similarity of $\mathbb{Z}_{-\beta}$, i.e. $-\beta \mathbb{Z}_{-\beta} \subset \mathbb{Z}_{-\beta}$, and on the following idea. Let $x=\gamma(w(d-1) \max (k))<y=\gamma(w d \min (k))$ be two neighbours in $\mathbb{Z}_{-\beta}$ with gap $\Delta_{k}$ and suppose only $k$ even. If we multiply both $x$ and $y$ by $(-\beta)$, we get a longer gap with possibly more $(-\beta)$ integers in between. It can be shown that between $-\beta y$ and $-\beta x$ there is always a gap $\Delta_{k+1}$. Hence the description is of the form $\Phi(k)=S_{k}(k+1) \widetilde{R_{k}}$, where the word $S_{k}$ codes the distances between $(-\beta)$ integers in $[\gamma(w d \min (k) 0), \gamma(w d \min (k+1))]$ and, similarly, $R_{k}$ encodes distances within the interval $[\gamma(w(d-1) \max (k) 0), \gamma(w(d-1) \max (k+1))]$.

As it turns out, in some cases (mostly when reference strings $l_{1} l_{2} l_{3} \cdots$ and $r_{1} r_{2} r_{3} \cdots$ are eventually periodic of a particular form) we can find a letter-to-letter projection to a finite alphabet $\Pi: \mathbb{N} \rightarrow \mathscr{B}$ with $\mathscr{B} \subset \mathbb{N}$, such that $\mathbf{u}_{-\beta}=\Pi \mathbf{v}_{-\beta}$ also encodes $\mathbb{Z}_{-\beta}$ and it is a fixed point of a an antimorphism $\varphi=\Pi \circ \Phi$ over the finite alphabet $\mathscr{B}$. Clearly, the square of $\varphi$ is then a non-erasing morphism over $\mathscr{B}$ which fixes $\mathbf{u}_{-\beta}$.

Let us mention that $(-\beta)$-integers in the Ito-Sadahiro case $l=-\frac{\beta}{\beta+1}$ are also subject of [8]. For $\beta$ with eventually periodic $d(l)$, Steiner finds a coding of $\mathbb{Z}_{-\beta}$ by a finite alphabet and shows, using only the properties of the $(-\beta)$-transformation, that the word is a fixed point of a non-trivial morphism. Our approach is of a combinatorial nature, follows a similar idea as in [1] and shows existence of an antimorphism for any base $\beta$.

To illustrate the results, let us conclude this contribution by an example.

Example 14. Let $\beta$ be the real root of $x^{3}-3 x^{2}-4 x-2(\beta$ Pisot, $\approx 4.3)$ and $l=-\frac{1}{2}$. The admissibility condition gives us for any admissible digit string $\left(x_{i}\right)_{i \geq 0}$ :

$$
201^{\omega} \preceq_{\text {alt }} x_{i} x_{i+1} x_{i+2} \cdots \prec_{\text {alt }} \overline{2} 0 \overline{1}^{\omega} \quad \text { for all } x \geq 0 .
$$

We obtain

$$
\min (0)=\varepsilon, \quad \min (1)=2, \quad \min (2)=20
$$


and

$$
\min (2 k+1)=20(11)^{k-1} 0, \quad \min (2 k+2)=20(11)^{k} \quad \text { for } k \geq 1 .
$$

Clearly it holds that $\max (i)=\overline{\min (i)}$ for all $i \in \mathbb{N}$.

Theorem 11 gives us the following distances within $\mathbb{Z}_{-\beta}$ :

$$
\Delta_{0}=1, \quad \Delta_{1}=-1+\frac{4}{\beta}+\frac{2}{\beta^{2}}, \quad \text { and } \quad \Delta_{2 k}=1-\frac{2}{\beta}-\frac{2}{\beta^{2}}, \quad \Delta_{2 k+1}=1+\frac{2}{\beta}+\frac{2}{\beta^{2}} \quad \text { for } k \geq 1 .
$$

Finally, the antimorphism $\Phi: \mathbb{N}^{*} \rightarrow \mathbb{N}^{*}$ is given by

$$
\begin{aligned}
& 0 \rightarrow 0^{2} 10^{2}, \\
& 1 \rightarrow 2, \\
& 2 \rightarrow 3,
\end{aligned}
$$

and for $k \geq 1$

$$
\begin{aligned}
& 2 k+1 \rightarrow 0^{2} 10(2 k+2) 010^{2}, \\
& 2 k+2 \rightarrow 2 k+3 .
\end{aligned}
$$

It can be easily seen that a projection from $\mathbb{N}$ to a finite alphabet exists and a final antimorphism $\varphi$ : $\{0,1,2,3\}^{*} \rightarrow\{0,1,2,3\}^{*}$ is of the form

$$
\begin{aligned}
& 0 \rightarrow 0^{2} 10^{2}, \\
& 1 \rightarrow 2 \\
& 2 \rightarrow 3 \\
& 3 \rightarrow 0^{2} 102010^{2} .
\end{aligned}
$$

\section{Bibliography}

[1] P. Ambrož, D. Dombek, Z. Masáková, E. Pelantová, Numbers with integer expansion in the numeration system with negative base, preprint (2011), 25pp. arXiv:0912.4597v3 [math.NT]

[2] D. Dombek, Z. Masáková, E. Pelantová, Number representation using generalized ( $\beta$ )-transformation, preprint (2011), 22pp. arXiv:1102.3079v1 [cs.DM]

[3] S. Fabre, Substitutions et $\beta$-systèmes de numération, Theoret. Comput. Sci. 137, 219-236 (1995). doi:10. 1016/0304-3975(95) 91132-A

[4] Ch. Frougny and A. C. Lai, On negative bases, Proceedings of DLT 09, Lectures Notes in Computer Science 5583 (2009). doi:10.1007/978-3-642-02737-6_20

[5] S. Ito and T. Sadahiro, Beta-expansions with negative bases, INTEGERS 9, 239-259 (2009). doi:10.1515/ INTEG.2009.023

[6] W. Parry, On the $\beta$-expansions of real numbers, Acta Math. Acad. Sci. Hung. 11, 401-416 (1960).

[7] A. Rényi, Representations for real numbers and their ergodic properties, Acta Math. Acad. Sci. Hung. 8, 477-493 (1957).

[8] W. Steiner, On the structure of ( $-\beta)$-integers, preprint (2010), 15pp. arXiv:1011.1755v1 [math.NT]

[9] W. P. Thurston, Groups, tilings, and finite state automata, AMS Colloquium Lecture Notes, American Mathematical Society, Boulder (1989). 